\title{
A FIXED POINT THEOREM FOR THE SUM OF TWO MAPPINGS
}

\author{
OLGA HADŽIĆC
}

\begin{abstract}
A generalization of a fixed point theorem of Rzepecki is proved and it is shown that in a paranormed space $E$ this result yields, under certain circumstances, solutions to the equation $x=T x+S x$ for $T: E \rightarrow E$ either continuous and affine or a generalized contraction, and $S: K \subseteq E \rightarrow E$ compact.
\end{abstract}

In [7] Zima proved a generalization of the Schauder fixed point theorem in a paranormed space setting. (Paranormed spaces are nonlocally convex topological vector spaces; see the definition below.) B. Rzepecki then proved the following generalization of Zima's result.

TheOREM 1 [6]. Let $X$ be a Hausdorff topological vector space, $K$ be a nonempty, closed and convex subset of $X$ and $T$ be a continuous mapping from $K$ into a compact set $Z(Z \subset K)$. Suppose that for every $x \in Z$ and every neighborhood $V$ of $x$ there exists a neighborhood $U$ of $x$ such that

$$
\operatorname{co}(U \cap Z) \subseteq V
$$

Then there exists $x \in K$ such that $x=T x$.

This is a generalization of Tihonov's fixed point theorem since we can suppose in the latter case, that $V$ is convex and so that $U=V$ in the above.

Let $E$ be a linear space over the real or complex number field. The function \|\| : $E \rightarrow[0, \infty)$ will be said to be paranormed iff:

1. $\|x\|^{*}=0 \Leftrightarrow x=0$.

2. $\|-x\|^{*}=\|x\|^{*}$, for every $x \in E$.

3. $\|x+y\|^{*} \leqslant\|x\|^{*}+\|y\|^{*}$, for every $x, y \in E$.

4. If $\left\|x_{n}-x_{0}\right\|^{*} \rightarrow 0$ and $\lambda_{n} \rightarrow \lambda_{0}$, then $\left\|\lambda_{n} x_{n}-\lambda_{0} x_{0}\right\|^{*} \rightarrow 0$.

The function $\rho: E \times E \rightarrow[0, \infty)$, defined by $\rho(x, y)=\|x-y\|^{*}(x, y \in E)$, is a distance function on $E$. If $(E, \rho)$ is a complete metric space, it is a Fréchet space. Furthermore $\left(E,\|\|^{*}\right)$ is a topological vector space and its family of neighborhoods of zero is given by $\left\{V_{\varepsilon}\right\}_{\varepsilon>0}$ where $V_{\varepsilon}=\left\{x \mid x \in E,\|x\|^{*}<\varepsilon\right\}$.

DEFINITION 1. Let $\left(E,\|\|^{*}\right)$ be a paranormed space and $K$ be a nonempty subset of $E$. We say that the set $K$ satisfies Zima's condition if there exists $C>0$ such that $\|\lambda x\|^{*} \leqslant C \lambda\|x\|^{*}$, for every $0 \leqslant \lambda \leqslant 1$ and every $x \in K-K$.

Received by the editors October 2, 1980 and, in revised form, March 13, 1981 and July 20, 1981. 1980 Mathematics Subject Classification. Primary 47H10.

Kel' words and phrases. Fixed point, paranormed space, $\Phi$ paranormed space, generalized contraction. 
Zima in [7] has given an example of a space $E$ and of a set $K$ which satisfies the above condition.

We now proceed to a generalization of Rzepecki's fixed point theorem. (We should point out, however, that our proof reduces to an application of Rzepecki's theorem.)

THEOREM 2. Let $X$ be a Hausdorff topological vector space, $K$ be a nonempty, closed and convex subset of $X, T: X \rightarrow X$ be an affine continuous mapping, and $S: K \rightarrow X$ be a continuous mapping such that $\overline{S(K)}$ is compact. Suppose that the following conditions are satisfied:

(i) For every $y \in \overline{c o} S(K)$ there exists one and only one solution $x(y) \in K$ of the equation $z=T z+y$ and the set $\{x(y)\}_{y \in \overline{S(K)}}$ is compact.

(ii) For every $V \in \mathcal{Q}$ and every $x \in \overline{S(K)}$ there exists $U \in \mathcal{Q}$ such that $\operatorname{co}((x+U) \cap \overline{S(K)}) \subseteq x+V$, where $\mathcal{Q}$ is the base of the neighborhoods of zero in $X$.

Then there exists $x \in K$ such that $x=T x+S x$.

Proof. We first prove that the mapping $R: y \rightarrow x(y)(y \in \overline{\mathrm{co}} S(K))$ is continuous on the set $\overline{S(K)}$. Suppose that $\left\{y_{\alpha}\right\}_{\alpha \in \mathcal{Q}}$ is a net from $\overline{S(K)}$ such that $\lim _{\alpha \in \mathbb{Q}} y_{\alpha}=y$ and such that, for every $\alpha \in \mathbb{Q}, R y_{\alpha}=T R y_{\alpha}+y_{\alpha}$. Since the set $\{x(y)\}_{y \in \overline{S(K)}}$ is compact, there exists a convergent subnet $\left\{R y_{\alpha_{\beta}}\right\}$ of the net $\left\{R y_{\alpha}\right\}$. Thus

$$
\lim _{\beta} R y_{\alpha_{\beta}}=T\left(\lim _{\beta} R y_{\alpha_{\beta}}\right)+\lim _{\beta} y_{\alpha_{\beta}}=T\left(\lim _{\beta} R y_{\alpha_{\beta}}\right)+y
$$

and so $\lim _{\beta} R y_{\alpha_{\beta}}$ is the solution of the equation $z=T z+y$, which implies that $\lim _{\beta} R y_{\alpha_{\beta}}=R y$. Since each subnet of the net $\left\{R y_{\alpha}\right\}$ has a convergent subnet with a limit $R y$, it follows that $\lim _{\alpha} R y_{\alpha}=R y$. It is obvious that $R^{-1}$ is continuous since

$$
R^{-1} z=z-T z \quad(z \in R(\overline{\operatorname{co}} S(K))) \text {. }
$$

Next we prove that the mapping $R$ is affine. Suppose that $\alpha, \beta \geqslant 0, \alpha+\beta=1$ and $x_{1}, x_{2} \in \overline{\operatorname{co}} \overline{S(K)}$. Then $R x_{1}=T R x_{1}+x_{1}, R x_{2}=T R x_{2}+x_{2}$ and so $\alpha R x_{1}+\beta R x_{2}$ $=T\left(\alpha R x_{1}+\beta R x_{2}\right)+\alpha x_{1}+\beta x_{2}$ which implies that $R\left(\alpha x_{1}+\beta x_{2}\right)=\alpha R x_{1}+$ $\beta R x_{2}$. Now, since $R$ is affine, for every convex set $M \subseteq \overline{c o} S(K)$ the set $R(M)$ is also convex. This implies that $R(\operatorname{co} N)$ is convex and so $\operatorname{co} R(\operatorname{co} N)=R(\operatorname{co} N)$. Since $R(N) \subseteq R(\operatorname{co} N)$ it follows that $\operatorname{co} R(N) \subseteq \operatorname{co} R(\operatorname{co} N)=R(\operatorname{co} N)$. We define the mapping $R^{*}: K \rightarrow K$ in the following way:

$$
R^{*} x=R S x, \text { for every } x \in K .
$$

We now show that the mapping $R^{*}$ satisfies all the conditions of Rzepecki's fixed point theorem, where the set $Z$ is taken to be the compact set $R(\overline{S(K)})$. Let $V \in \mathcal{Q}$ and $x \in R(\overline{S(K)})$. We shall prove that there exists $U \in \mathcal{Q}$ such that

$$
\operatorname{co}((x+U) \cap R(\overline{S(K)})) \subseteq x+V, \text { for every } x \in R(\overline{S(K)}) .
$$

Since $x \in R(\overline{S(K)})$, there exists $u \in \overline{S(K)}$ such that $x=R u$. The mapping $R$ is continuous at the point $u$ and so there exists $V^{\prime} \in \mathcal{Q}$ such that

$$
R\left(\left(u+V^{\prime}\right) \cap \overline{\operatorname{co}} S(K)\right) \subseteq R u+V
$$


Furthermore, from (ii) it follows that there exists $U^{\prime} \in \mathcal{U}$ such that

$$
\operatorname{co}\left(\left(u+U^{\prime}\right) \cap \overline{S(K)}\right) \subseteq u+V^{\prime} .
$$

From (1) it follows that

$$
R\left(\operatorname{co}\left(\left(u+U^{\prime}\right) \cap \overline{S(K)}\right)\right) \subseteq R\left(\left(u+V^{\prime}\right) \cap \overline{\operatorname{co}} S(K)\right) \subseteq R u+V
$$

and since $R$ is a one-to-one mapping

$$
\operatorname{co}\left(R\left(u+U^{\prime}\right) \cap R(\overline{S(K)})\right) \subseteq R u+V .
$$

The mapping $R^{-1}$ is continuous, and so there exists $U \in \mathcal{U}$ such that

$$
R^{-1}(R u+U) \subseteq R^{-1}(R u)+U^{\prime}=u+U^{\prime},
$$

and thus $(R u+U) \cap R(\overline{S(K)}) \subseteq R\left(\left(u+U^{\prime}\right) \cap(\overline{S(K)})\right)$. From (2) we conclude that

$$
\operatorname{co}((R u+U) \cap R(\overline{S(K)})) \subseteq R u+V 4
$$

and so the mapping $R^{*}$ satisfies all the conditions of Theorem 1 . This implies that $\operatorname{Fix}\left(R^{*}\right) \neq \varnothing$ and, since $\operatorname{Fix}\left(R^{*}\right) \subseteq \operatorname{Fix}(T+S)$, it follows that $\operatorname{Fix}(T+S) \neq \varnothing$.

Corollary 1. Let $\left(E,\|\|^{*}\right)$ be a paranormed space and $K$ be a nonempty, closed and convex subset of $E$. Let $T: E \rightarrow E$ be a continuous and affine mapping, $S: K \rightarrow E$ be a continuous mapping such that $\overline{S(K)}$ is compact and satisfies Zima's conditions, and suppose for every $y \in \overline{c o} S(K)$ there exists one and only one solution $x(y) \in K$ of the equation $z=T z+y$ with $\{x(y)\}_{y \in \overline{S(K)}}$ compact. Then there exists $x \in K$ such that $x=T x+S x$.

Proof. It is easy to see that, since $\overline{S(K)}$ satisfies Zima's condition, the condition (ii) of Theorem 2 is satisfied and so there exists $x \in E$ such that $x=T x+S x$.

Definition 2. [5] Let $(X, d)$ be a metric space and $T: X \rightarrow X$. The mapping $T$ : $X \rightarrow X$ is a generalized contraction iff $d(T x, T y) \leqslant L(r, s) d(x, y)$, for every $x, y \in X$, $r \leqslant d(x, y) \leqslant s$, where the function $L$ is defined for every $(r, s) \in(0, \infty) \times(0, \infty)$ such that $r \leqslant s$ and $L(r, s)<1$.

REMARK. The fixed point theorem of [5] for generalized contractions, which we use below, is also an immediate consequence of the fixed point theorem of A. Meir and E. Keeler [4].

From Corollary 1 we can derive the following corollary.

COROLlARY 2. Let $\left(E,\|\|^{*}\right)$ be a paranormed space, $K$ be a nonempty, convex and complete subset of $E, T: E \rightarrow E$ be an affine generalized contraction mapping, $S$ : $K \rightarrow E$ be a compact mapping such that $T(K)+\overline{c o} S(K) \subseteq K$, the set $\overline{S(K)}$ satisfies Zima's condition and the set $(I-T)^{-1} \overline{S(K)}$ be bounded. Then there exists $x \in K$ such that $x=T x+S x$.

Proof. Since $T(K)+\overline{c o} S(K) \subseteq K$ and $T$ is generalized contraction for each $y \in \overline{c o} S(K)$ there exists one and only one element $R y \in K$ such that $R y=T R y+y$ ([5]; cf. [4]). It remains to be proved that the set $\{R y\}_{y \in \overline{S(K)}}$ is compact. To do this we shall show that the mapping $R$ is continuous. Suppose that $\left\{x_{n}\right\}_{n \in N} \subseteq \overline{S(K)}$ and 
that $\lim _{n \rightarrow \infty} x_{n}=x$. If, on the contrary, the mapping $R$ is not continuous, then there exists $\varepsilon>0$ and a sequence $\{n(k)\}_{k \in N} \subseteq N$ such that

$$
\left\|R x_{n(k)}-R x\right\|^{*} \geqslant \varepsilon \quad(n(k) \geqslant k, \text { for every } k \in N) .
$$

Since the set $(I-T)^{-1} \overline{S(K)}$ is bounded, there exists $K^{\prime}>0$ such that $\|R y\|^{*} \leqslant K^{\prime}$, for every $y \in \overline{S(K)}$, and so for every $k \in N$,

$$
\left\|R x_{n(k)}-R x\right\|^{*} \leqslant 2 K^{\prime} .
$$

This in turn implies that

(3) $\left\|R x_{n(k)}-R x\right\|^{*} \leqslant L\left(\varepsilon, 2 K^{\prime}\right)\left\|R x_{n(k)}-R x\right\|^{*}+\left\|x_{n(k)}-x\right\|^{*}, \quad k \in N$.

Since $\left\{\left\|R x_{n(k)}-R x\right\|^{*} \mid k \in N\right\} \subseteq\left[\varepsilon, 2 K^{\prime}\right]$, there exists a subsequence $\left\{x_{n(k(r))}\right\}_{r \in N}$ such that

$$
m=\lim _{r \rightarrow \infty}\left\|R x_{n(k(r))}-R x\right\|^{*}
$$

and so, from (3), we have

$$
m \leqslant L\left(\varepsilon, 2 K^{\prime}\right) m<m
$$

which is a contradiction.

We shall now give an application of Theorem 2 which refers to the existence of a solution to the equation $x=T x+S x$ in $\Phi$-paranormed spaces [2]. We begin with some notations and definitions. We shall subsequently denote the set of all real numbers by $R$. Furthermore, let $E$ be a vector space over $\mathcal{K}$ (real or complex number field) and $R_{\Delta}$ be the set of all mappings from $\Delta$ into $R$. The Tihonov product topology and the operations of + and scalar multiplication are as usual. If $f, g \in R$ we say that $f \leqslant g$ iff $f(t) \leqslant g(t)$, for every $t \in \Delta$, and by $P_{\Delta}$ we shall denote the cone of nonnegative elements in $R_{\Delta}$.

In [2] $\mathrm{S}$. Kasahara introduced the following notion of paranormed spaces, which we shall call a $\Phi$ paranormed space.

Definition 3. The triplet $(E,\|\|, \Phi)$ is a $\Phi$ paranormed space iff \|\|$: E \rightarrow P_{\Delta}$ and $\Phi$ is a linear, continuous, positive mapping from $R_{\Delta}$ into $R_{\Delta}$ such that the following conditions are satisfied:

1. $\|x\|=0 \Leftrightarrow x=0$.

2. $\|\lambda x\|=|\lambda|\|x\|$, for every $x \in E$ and every $\lambda \in \mathscr{K}$.

3. $\|x+y\| \leqslant \Phi(\|x\|)+\Phi(\|y\|)$, for every $x, y \in E$.

Let $\mathscr{U}$ denote the family of neighborhoods of zero in $R_{\Delta}$. For each $U \in \mathcal{Q}$ we denote the set $\{x \mid x \in E,\|x\| \in U\}$ by $V_{U}$. Then $E$ is a topological vector space in which $\left\{V_{U}\right\}_{U \in \mathcal{Q}}$ is the family of neighborhoods of zero in $E$.

In [2] it is proved that every Hausdorff topological vector space is a $\Phi$ paranormed space $(E,\|\|, \Phi)$ over a topological semifield $R_{\Delta}$.

Definition 4. Let $(E,\|\|, \Phi)$ be a $\Phi$ paranormed space over a topological semifield $R_{\Delta}$ and $K \subseteq E$. If for every $n \in N$, every $u_{i} \in K-K(i=1,2, \ldots, n)$ and $\left(s_{1}, s_{2}, \ldots, s_{n}\right) \in R^{n}$ such that $s_{i} \in[0,1](i=1,2, \ldots, n)$ and $\sum_{i=1}^{n} s_{i}=1$,

$$
\left\|\sum_{i=1}^{n} s_{i} u_{i}\right\| \leqslant \sum_{i=1}^{n} s_{i} \Phi\left(\left\|u_{i}\right\|\right),
$$

we say that the set $K$ is of $\Phi$-type. 
In [3] Matusov used Kasahara's result in order to prove a fixed point theorem.

Let $\mathcal{U}$ be the family of neighborhoods of zero in $R_{\Delta}$ and $U \in \mathcal{U}$. Then $\{x \mid x \in E$, $\|x\| \in U\}$ is a neighborhood of zero $V_{U}$ in $E$ and let us denote the family $\left\{V_{U}\right\}_{U \in \mathcal{U}}$ by $U^{\prime}$. Suppose now that $K$ is a subset of $E$ and that $K$ is of $\Phi$-type. We prove that for every $V \in \mathcal{U}^{\prime}$ there exists $V^{\prime} \in \mathcal{U}^{\prime}$ such that for every $x \in K$

$$
\operatorname{co}\left(\left(x+V^{\prime}\right) \cap K\right) \subseteq x+V \text {. }
$$

Since $V \in \mathcal{Q}^{\prime}$, there exists $\mu=\left\{t_{1}, t_{2}, \ldots, t_{n}\right\} \subseteq \Delta$ and $\varepsilon>0$ such that

$$
\|u\| \in U_{\mu, \varepsilon} \Rightarrow u \in V
$$

where $U_{\mu, \varepsilon}=\{x \mid\|x\|(t)<\varepsilon$, for every $t \in \Delta\}$. Since the mapping $\Phi$ is linear and continuous there exists $V^{\prime}=V_{U}$, such that

$$
u \in V^{\prime} \Rightarrow \Phi(\|u\|) \in U_{\mu, \varepsilon} \text {. }
$$

It is easy to see that

$$
\operatorname{co}\left(\left(x+V^{\prime}\right) \cap K\right) \subseteq x+V, \text { for every } x \in K .
$$

Indeed, suppose that $u \in \operatorname{co}\left(\left(x+V^{\prime}\right) \cap K\right)$. Then $u=\sum_{i=1}^{n} \lambda_{i} x_{i}$ where $x_{i} \in(x+$ $\left.V^{\prime}\right) \cap K(i=1,2, \ldots, n), \lambda_{i} \geqslant 0(i=1,2, \ldots, n)$ and $\sum_{i=1}^{n} \lambda_{i}=1$. Thus

$$
\|u-x\|(t)=\left\|\sum_{i=1}^{n} \lambda\left(x_{i}-x\right)\right\|(t) \leqslant \sum_{i=1}^{n} \lambda_{i} \Phi\left(\left\|x_{i}-x\right\|\right)(t) \leqslant \varepsilon,
$$

for every $t \in \mu$, and so $\|u-x\| \in U_{\mu, \varepsilon}$. This implies that $u-x \in V$ and so $u \in x+V$.

Now, we can formulate the following corollary.

Corollary 3. Let $(X,\|\|, \Phi)$ be a $\Phi$-paranormed space, $K$ be a nonempty, closed and convex subset of the space $X, T: X \rightarrow X$ be an affine continuous mapping, and $S$ : $K \rightarrow X$ be a continuous mapping such that $\overline{S(K)}$ is compact and of $\Phi$-type. Suppose also that the condition (i) of Theorem 2 is satisfied. Then there exists $x \in K$ such that $x=T x+S x$.

\section{REFERENCES}

1. O. Hadžiç, Fixed point theorems for multivalued mappings in topological vector spaces, Glas. Mat. Ser. III 14 (33) (1980), 113-120.

2. S. Kasahara, On formulations of topological linear spaces by topological semifields, Math. Japon. 19 (1974), 121-134.

3. V. E. Matusov, Obobshcheniye teoremii o nepodvizhnoi tochke Tikhonova, Dokl. Akad. Nauk UzSSR 1978, no. 2, 12-14.

4. A. Meir and E. Keeler, A theorem on contraction mappings, J. Math. Anal. Appl. 28 (1969), 326-329.

5. T. Riedrich, Vorlesungen über nichtlineare Operatoren-gleichungen, Teubner, Leipzig, 1976.

6. B. Rzepecki, Remarks on Schauder's Fixed Point Theorem, Bull. Acad. Polon. Sci. Sér. Sci. Math. Astronom. Phys. 27 (1976), 473-480.

7. K. Zima, On the Schauder's fixed point theorem with respect to paranormed space, Comment. Math. Prace Mat. 19 (1977), $421-423$.

Department of Mathematics, University of Novi Sad, Faculty of Sciences, 21000 Novi Sad, ulica Dr Ilije Djuricica 4, Yugoslavia 